\title{
Electromagnetic Response in Kinetic Energy Driven Superconductivity: the Meissner Effect
}

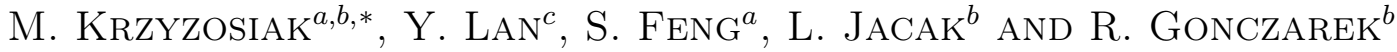 \\ ${ }^{a}$ Department of Physics, Beijing Normal University, Beijing 100875, China \\ ${ }^{b}$ Institute of Physics, Wrocław University of Technology \\ Wybrzeże Wyspiańskiego 27, 50-370 Wrocław, Poland \\ ${ }^{c}$ Department of Physics, Jinan University, Guangzhou 510623, China
}

\begin{abstract}
Electromagnetic response of cuprate superconductors is studied within the model of kinetic energy driven $d$-wave superconductivity by analyzing the Meissner effect. The kernel of the linear response function is found and employed to calculate the magnetic field penetration depth and the superfluid density of cuprate superconductors within the specular reflection model for a purely transverse vector potential. It is shown that the magnetic field penetration depth and the superfluid density depend linearly on temperature, except for a strong deviation from the linear characteristics at extremely low temperatures, which is attributed to nonlocal effects. The zero-temperature superfluid density is found to decrease linearly with decreasing doping concentration in the underdoped regime. The problem of gauge invariance in the theoretical description of the electromagnetic response is addressed, and an approximation which does not violate local charge conservation is proposed.
\end{abstract}

PACS numbers: 74.25.Ha, 74.25.N-, 74.20.Mn

\section{Introduction}

The spectacular Meissner-Ochsenfeld effect in which the external magnetic field is expelled from a superconducting (SC) sample is one of the most fundamental phenomena observed in superconductors, and can be used to infer about many fundamental features of the studied system. Therefore the physics of superconductors at the length scale of the penetration depth, i.e. the region at the edge of the sample where the induced supercurrents effectively screen the external magnetic field, has been intensively studied by both theoretical and experimental methods in high- $T_{\mathrm{c}}$ superconductivity research.

It is the linear low temperature dependence of the magnetic field penetration depth $\lambda(T)$ which first provided a strong experimental support for nodes in the SC order parameter of cuprate superconductors [1], then confirmed by angle resolved photoemission spectroscopy (ARPES) [2]. The linear temperature dependence of the penetration depth observed in various SC cuprates $[1,3]$ is characteristic of the $d$-wave $\mathrm{SC}$ energy gap and is attributed to excitation of quasiparticles (QPs) out of the condensate at the nodes of the order parameter. On the other hand, the doping dependence of the electromagnetic response in cuprate superconductors can be studied in terms of the zero-temperature superfluid density. The

* corresponding author; e-mail: mateusz.krzyzosiak@pwr.wroc.pl superfluid density, which is proportional to the squared amplitude of the coherent macroscopic wave function of the SC charge carriers, can provide significant information about the SC state. The zero-temperature superfluid density in cuprate superconductors is found to decrease linearly with decreasing doping [4], which in turn implies a linear relation between the critical temperature $T_{\mathrm{c}}$ and the superfluid density observed in underdoped samples [5].

The value of the magnetic field penetration depth in cuprate superconductors is much larger than the coherence length regarded as a measure of the spatial extent of a Cooper pair. Consequently, cuprate superconductors can be regarded as type-II superconductors and the local, London-type electrodynamics should be applicable, resulting in an exponential decay of the local magnetic field inside the sample $[6,7]$. Nonlocal effects in superconductors emerge when the electromagnetic field varies significantly over the size of a Cooper pair. It has been suggested [8] that nonlocal effects can also imply a crossover in the low-temperature dependence of the penetration depth even in the clean limit. This is because the effective coherence length diverges at the nodes, and so the locality condition based on the Ginzburg-Landau ratio of the penetration depth and the coherence length no longer holds [8].

The layered structure of cuprate superconductors gives rise to strong anisotropy, and it is possible to observe both the in-plane and the inter-plane Meissner effects. 
The former one is characterized by the $a b$-plane magnetic field penetration depth, whereas the latter one is related to the magnetic field penetration in the $c$-axis direction. Here we concentrate on the in-plane Meissner effect based on the kinetic energy driven superconductivity $[9,10]$ and do not consider $c$-axis properties, which can be discussed, e.g., by taking into account hopping between adjacent copper-oxides layers within the tunneling Hamiltonian approach [11].

\section{Model and method}

It has been argued that the physics of the doped $\mathrm{CuO}_{2}$ plane $[2,12]$ can be described by the $t-J$ model [13] on a square lattice, which in the present case needs to be extended by including the exponential Peierls factors

$$
\begin{aligned}
H & =-t \sum_{l \hat{\eta} \sigma} \mathrm{e}^{-\mathrm{i}(e / \hbar) \boldsymbol{A}(l) \cdot \hat{\eta}} C_{l \sigma}^{\dagger} C_{l+\hat{\eta} \sigma}+\mu \sum_{l \sigma} C_{l \sigma}^{\dagger} C_{l \sigma} \\
& +J \sum_{l \hat{\eta}} \boldsymbol{S}_{l} \cdot \boldsymbol{S}_{l+\hat{\eta}} .
\end{aligned}
$$

Here $\hat{\eta}= \pm \hat{x}, \pm \hat{y}, C_{l \sigma}^{\dagger}\left(C_{l \sigma}\right)$ is the electron creation (annihilation) operator, $\boldsymbol{S}_{l}=\left(S_{l}^{x}, S_{l}^{y}, S_{l}^{z}\right)$ are spin operators, $\mu$ is the chemical potential, and the Peierls factors account for the coupling of electrons to the external magnetic field represented by the vector potential $\boldsymbol{A}(\boldsymbol{r})$. The extended $t-J$ Hamiltonian (1) is subjected to an important local constraint $\sum_{\sigma} C_{l \sigma}^{\dagger} C_{l \sigma} \leq 1$ in order to avoid the double occupancy. The strong electron correlation manifests itself by this local constraint [12], which can be treated properly in analytical calculations within the charge-spin separation fermion-spin theory [14], where the constrained electron operators are decoupled as $C_{l \uparrow}=h_{l \uparrow}^{\dagger} S_{l}^{-}$and $C_{l \downarrow}=h_{l \downarrow}^{\dagger} S_{l}^{+}$. The spinful fermion operator $h_{l \sigma}=\mathrm{e}^{-\mathrm{i} \Phi_{l \sigma}} h_{l}$ represents the charge degree of freedom together with some effects of spin configuration rearrangements due to the presence of the doped hole itself (charge carrier), while the spin operator $S_{l}$ represents the spin degree of freedom (spin). Then the electron single occupancy local constraint is satisfied automatically [14].

It is worth to emphasize that in general, due to renormalization related to the reduction of the energy scale, the kinetic energy and the potential energy at the $t-J$ model level become mixed [15, 16]. Therefore the observation that the hopping energy defined for the $t-J$ model is lowered, does not mean that the kinetic energy defined from the first principles is lowered. The kinetic energy defined in the parent model is not identical with the kinetic energy in the model derived from it by applying a unitary transformation as discussed in Ref. [15]. However, if the system manages somehow to simultaneously lower the hopping energy and the exchange energy at the $t-J$ model level, the decrease in the total and kinetic energies defined at the Hubbard model level is then an obvious consequence [15]. The lowering of the latter one in the Hubbard model in the superconducting state contradicts experiments, indicating that a single band model is not able to describe the excitations across the gap properly. However, an effective model - such as the $t-J$ model - captures the essential physics of low-energy excitations [15].

Although the external magnetic field usually represents a large perturbation, it is cancelled over most of the volume of the sample by the induced field generated by the supercurrents. Consequently, the net field acts only very near the surface on a scale of the magnetic field penetration depth and so it can be treated as a weak perturbation. The Meissner effect can be therefore successfully studied within the linear response approach [17, 18], where the averaged value $\boldsymbol{J}$ of the microscopic screening current is found as

$$
J_{\mu}(\boldsymbol{q}, \omega)=-\sum_{\nu} K_{\mu \nu}(\boldsymbol{q}, \omega) A_{\nu}(\boldsymbol{q}, \omega) .
$$

Here $K_{\mu \nu}=K_{\mu \nu}^{(\mathrm{d})}+K_{\mu \nu}^{(\mathrm{p})}$ is the kernel of the response function, and once known allows us to calculate quantitative characteristics of the electromagnetic response.

The charge current operator, obtained e.g. as a functional derivative of the Hamiltonian (1) with respect to to the vector potential [19] includes a diamagnetic (d) and a paramagnetic (p) component. Since the former one is explicitly proportional to the vector potential, the diamagnetic contribution to the kernel can be found from Eq. (2) as [20]:

$$
\begin{aligned}
& K_{\mu \nu}^{(\mathrm{d})}(\boldsymbol{q})=-\frac{Z_{h F} \chi e^{2} t}{2 \hbar^{2} N} \\
& \quad \times \sum_{\boldsymbol{k}} \delta_{\mu \nu}\left(1-\frac{\bar{\xi}_{\boldsymbol{k}}}{E_{h \boldsymbol{k}}} \tanh \frac{\beta E_{h \boldsymbol{k}}}{2}\right) \cos k_{\mu},
\end{aligned}
$$

where $\chi$ is a spin correlation function, $Z_{h F}$ is the QP coherence factor and $E_{h \boldsymbol{k}}=\sqrt{\bar{\xi}_{\boldsymbol{k}}^{2}+\bar{\Delta}_{h Z}(\boldsymbol{k})^{2}}$ is the QPs spectrum $[9,10]$. On the other hand, the paramagnetic contribution can only be calculated approximately as it involves evaluation of a retarded current-current correlation function (polarization bubble). Here we will restrict the discussion to evaluation - within the Matsubara formalism - of the polarization bubble with bare paramagnetic current vertices $\gamma$, but dressed charge carrier Green functions $\mathbb{G}$ as shown in Fig. 1. It is well known [21] that employing this scenario we do not take into account longitudinal excitations properly, and this approach is valid

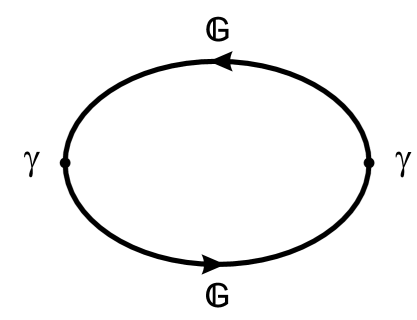

Fig. 1. Bare polarization bubble: solid lines represent the charge carrier Green functions in the Nambu notation [14]. 
for the vector potential in a purely transverse gauge only, such as the Coulomb gauge. If we would like to keep the theory gauge invariant, we would have to dress the polarization bubble in a way which maintains the generalized Ward identity [21], e.g., by adapting a ladder-type approximation for the current vertex. Such dressing is briefly discussed in Appendix at the end of the paper.

In the static limit $(\omega \approx 0)$ the bare vertex paramagnetic part of the response kernel can be found as [20]:

$$
\begin{aligned}
& K_{\mu \nu}^{(\mathrm{p})}(\boldsymbol{q}, 0)=-\left(\frac{\chi e t Z_{h F}}{N \hbar^{2}}\right)^{2} \mathrm{e}^{\frac{\mathrm{i}}{2}\left(q_{\mu}-q_{\nu}\right)} \sum_{\boldsymbol{k}} \sin \left(k_{\mu}+\frac{q_{\mu}}{2}\right) \sin \left(k_{\nu}+\frac{q_{\nu}}{2}\right) \\
& \quad \times\left\{\frac{1}{E_{h \boldsymbol{k}}+E_{h \boldsymbol{k}+\boldsymbol{q}}}\left[1-\frac{\bar{\xi}_{\boldsymbol{k}+\boldsymbol{q}} \bar{\xi}_{\boldsymbol{k}}+\bar{\Delta}_{h Z}(\boldsymbol{k}+\boldsymbol{q}) \bar{\Delta}_{h Z}(\boldsymbol{k})}{E_{h \boldsymbol{k}} E_{h \boldsymbol{k}+\boldsymbol{q}}}\right]\left[1-n_{\mathrm{F}}\left(E_{h \boldsymbol{k}}\right)-n_{\mathrm{F}}\left(E_{h \boldsymbol{k}+\boldsymbol{q}}\right)\right]\right. \\
& \left.+\frac{1}{E_{h \boldsymbol{k}}-E_{h \boldsymbol{k}+\boldsymbol{q}}}\left[1+\frac{\bar{\xi}_{\boldsymbol{k}+\boldsymbol{q}} \bar{\xi}_{\boldsymbol{k}}+\bar{\Delta}_{h Z}(\boldsymbol{k}+\boldsymbol{q}) \bar{\Delta}_{h Z}(\boldsymbol{k})}{E_{h \boldsymbol{k}} E_{h \boldsymbol{k}+\boldsymbol{q}}}\right]\left[n_{\mathrm{F}}\left(E_{h \boldsymbol{k}+\boldsymbol{q}}\right)-n_{\mathrm{F}}\left(E_{h \boldsymbol{k}}\right)\right]\right\},
\end{aligned}
$$

where $n_{\mathrm{F}}$ is the Fermi function. Let us note that in the long wavelength limit, when $|\boldsymbol{q}| \rightarrow 0$, the former term in Eq. (3) vanishes, and the latter reduces to $-2\left(\chi e t Z_{h F} / N \hbar^{2}\right)^{2} \sum_{\boldsymbol{k}} \sin k_{\mu} \sin k_{\nu} n_{\mathrm{F}}\left(E_{h \boldsymbol{k}}\right)\left[1-n_{\mathrm{F}}\left(E_{h \boldsymbol{k}}\right)\right]$, which is equal to zero in the zero-temperature limit. Hence, in this case, the long wavelength Meissner effect at $T=0$ is determined by the diamagnetic part of the kernel only.

\section{Results and discussion}

The reaction of the system to a weak electromagnetic stimulus is entirely described by the linear response kernel calculated within an assumed microscopic model. Technically, we need to combine one of the Maxwell equations with the linear relation between the current and the vector potential describing the response of the system, and solve them together for the vector potential. This is the step in which a particular gauge of the vector potential is set. We take into account the confined geometry of the cuprates by employing the standard specular reflection model [22] with a two-dimensional geometry of the SC plane, in the configuration with external magnetic field perpendicular to the $a b$ plane. In the assumed geometry the in-plane magnetic field penetration depth can be found as [20]:

$$
\lambda(T)=\frac{2}{\pi} \int_{0}^{\infty} \frac{\mathrm{d} q_{x}}{\mu_{0} K_{y y}\left(q_{x}, 0,0\right)+q_{x}^{2}} .
$$

The zero-temperature value of the penetration depth $\lambda(0) \approx 380.8 \mathrm{~nm}$ for $\delta=0.15$ and $t / J=2.5$ (with a reasonably estimative value of $J / k_{\mathrm{B}} \approx 1000 \mathrm{~K}$ ) is in the range $\lambda(0) \approx 156 \div 400 \mathrm{~nm}$ observed in different families of cuprate superconductors [6]. Our theoretical result for $\Delta \lambda(T)$ presented in Fig. 2 clearly shows linearity in the temperature, except for extremely low temperatures where a strong deviation from the linear characteristics appears. This crossover from the linear temperature dependence into the nonlinear one is observed experimentally in nominally clean cuprate superconductors $[3,6,23,24]$. Having calculated the penetration depth we can easily find the superfluid density at zero temperature (Fig. 3), and note that it decreases linearly with decreasing doping level. This result also is a natural consequence of the linear doping dependence of the SC transition temperature $T_{\mathrm{c}} \propto \delta$ found in the underdoped regime within the framework of the kinetic energy driven SC mechanism $[9,10]$ : the SC transition temperature is set by the charge carrier doping concentration, and then the density of the charge carriers directly determines the in-plane superfluid density in the underdoped regime. The temperature dependence of superfluid density is presented in Fig. 4 and also stays in agreement with experimental observations [4].

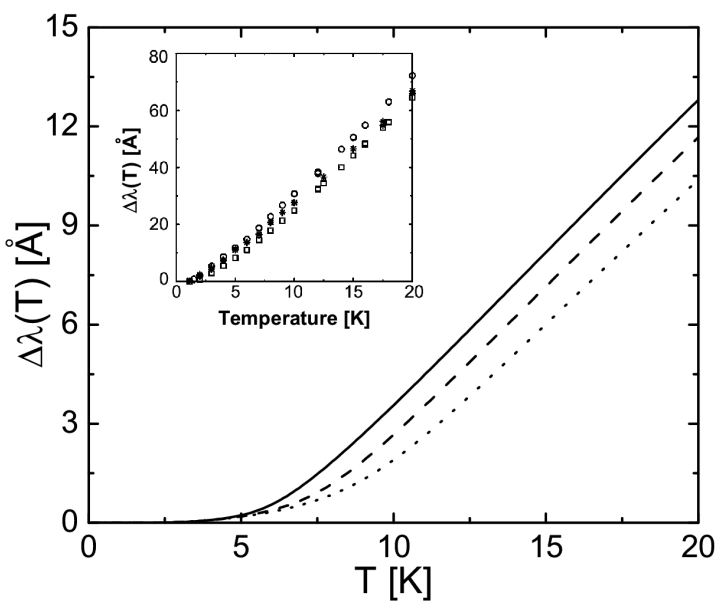

Fig. 2. Temperature dependence of magnetic field in-plane penetration depth $\Delta \lambda(T)$ for the doping concentration $\delta=0.150$ (solid), 0.149 (dashed), and 0.148 (dotted) with parameter $t / J=2.5$. Inset: the experimental result for $\mathrm{YBa}_{2} \mathrm{Cu}_{3} \mathrm{O}_{7-y}$ (data taken from [3]).

The nonlinearity observed in the temperature dependence of the penetration depth (Fig. 2) and the superfluid density (Fig. 4) can be attributed to nonlocal effects, which in the case of a pure $d$-wave cuprate supercon- 


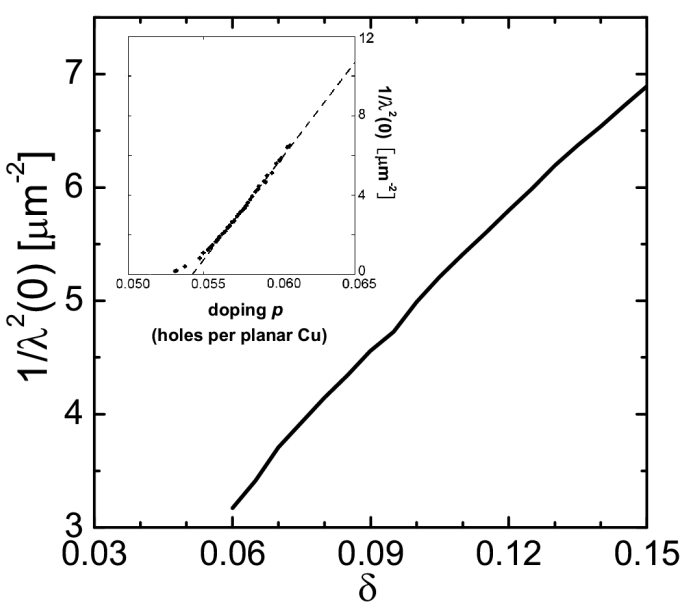

Fig. 3. Doping dependence of the zero-temperature in-plane superfluid density in the underdoped regime with $t / J=2.5$ for the doping concentration $\delta=0.072$ (solid line), $\delta=0.069$ (dashed line), and $\delta=0.066$ (dotted line). Inset: the corresponding experimental results for $\mathrm{YBa}_{2} \mathrm{Cu}_{3} \mathrm{O}_{7-y}$ taken from Ref. [4].

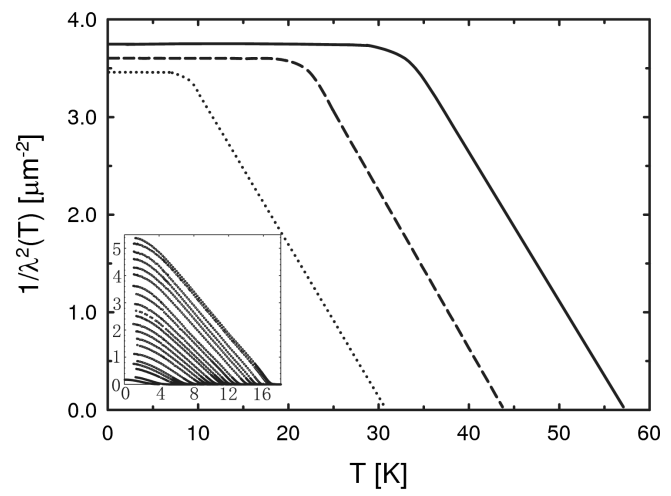

Fig. 4. Temperature dependence of the in-plane superfluid density for the doping concentration $\delta=0.072$ (solid line), $\delta=0.069$ (dashed line), and $\delta=0.066$ (dotted line) with $t / J=2.5$. Inset: the corresponding experimental results for $\mathrm{YBa}_{2} \mathrm{Cu}_{3} \mathrm{O}_{7-y}$ taken from Ref. [4].

ductor may become significant in the electromagnetic response [8]. In general, the relation between the supercurrent and the vector potential is nonlocal in the coordinate space due to the finite size of charge carrier Cooper pairs, which in the clean limit is of the order of the coherence length $\zeta(\boldsymbol{k})=\hbar v_{\mathrm{F}} / \pi \Delta_{h}(\boldsymbol{k})$ and is momentum dependent. Here $v_{\mathrm{F}}=\hbar^{-1} \partial \xi_{\boldsymbol{k}} /\left.\partial \boldsymbol{k}\right|_{k_{\mathrm{F}}}$ is the charge carrier velocity at the Fermi surface. Although the external magnetic field decays exponentially on the scale of the magnetic field in-plane penetration length $\lambda(T)$, nonlocal contributions to measurable quantities are of the order of $\kappa^{-2}$, where $\kappa=\lambda / \zeta$ is known as the Ginzburg-Landau (GL) parameter. In the $d$-wave cuprate superconductors, the characteristic feature is the existence of four nodal points in the Brillouin zone, where the charge carrier gap function vanishes and hence the coherence length $\zeta(\boldsymbol{k})$ diverges. At extremely low temperatures the QPs selectively populate the nodal regions, and the major contribution to measurable quantities comes from these QPs. In these conditions, the GL ratio is no longer large enough for the system to belong to the class of type-II superconductors, and the condition of the local limit is not fulfilled [8]. The system falls then into the extreme nonlocal limit, and the nonlinear characteristics in the temperature dependence of the penetration depth can be observed at sufficiently low temperatures $[6,23]$. However, with increasing temperature, the QPs around the nodal regions become excited out of the condensate, and the nonlocal effect fades away. In this case, the momentum dependent coherence length can be replaced approximately with the isotropic one $\zeta_{0}=\hbar v_{\mathrm{F}} / \pi \Delta_{h}$. Then the GL parameter $\kappa_{0} \approx \lambda(0) / \zeta_{0} \approx 180$, and the condition for the local limit is satisfied. This value of the GL parameter falls into the range $\kappa_{0} \approx 150 \div 400$ estimated experimentally for various cuprate superconductors [6]. Consequently, the cuprate superconductors at moderately low temperatures turn out to be type-II superconductors, where nonlocal effects are negligible and the electrodynamics is purely local. In this local limit, the pure $d$-wave pairing state in the kinetic energy driven SC mechanism $[9,10]$ gives the magnetic field penetration depth $\Delta \lambda(T) \propto T[8]$.

\section{Summary}

In this paper we have discussed the electromagnetic response of cuprate superconductors by analyzing the expulsion of the external magnetic field in the MeissnerOchsenfeld effect. Within the framework of kinetic energy driven $d$-wave superconductivity, following the linear response theory, and taking into account the two-dimensional geometry of the copper-oxide planes within the specular reflection model, we have reproduced some features of the electromagnetic response experiments on cuprate superconductors, including the linear temperature characteristics of the in-plane penetration depth and the superfluid density in the low temperature regime, and their nonlinear temperature dependence at extremely low temperatures. We have also found the doping evolution of the zero temperature superfluid density, which turned out to be linear for underdoped systems. In the paper, we have also emphasized the limitations of the used approximations, especially with respect to the problem of gauge invariance.

\section{Acknowledgments}

The authors would like to thank Zhi Wang for helpful discussions. This work was supported by the National Natural Science Foundation of China and the Ministry of Science and Technology of China. M.K. gratefully acknowledges financial support from a research scholarship funded by Institute of Physics, Wrocław University of Technology. 


\section{Appendix: gauge-invariant polarization bubble}

Gauge invariance is a direct consequence of local charge conservation [18, 21], which mathematically is expressed by the charge density-current continuity equation. In the Green function formalism the continuity equation turns into its Green function analogue called the generalized Ward identity (GWI) [18, 21, 25, 26]. Since the local charge conservation is a quite universal and fundamental principle, it should be inherent to any theory of the Meissner-Ochsenfeld effect which is expected to be gauge invariant. Here - within the formalism of kinetic energy driven superconductivity $[9,10]$ — we outline [20] a method to dress the current vertex in a way, which does not violate the GWI. Once this method is applied, the bare polarization bubble (Fig. 1) can be replaced with its dressed version presented in Fig. 5 and the resulting kernel of the response function will provide correct results for any gauge of the vector potential.

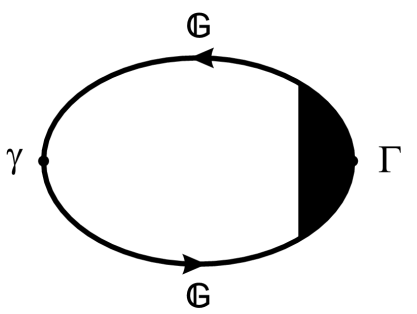

Fig. 5. Dressed polarization bubble: the solid lines represent the charge carrier Green functions in the Nambu notation.

It is well known that in order to obtain a dressed vertex function, which does not violate the GWI, a ladder-type approximation can be adapted $[18,21,25]$. The nature of the pairing mechanism in kinetic energy driven superconductivity, which originates from the so-called spin bubble $[9,10]$, suggests a ladder-like approximation of the form presented in Fig. 6.

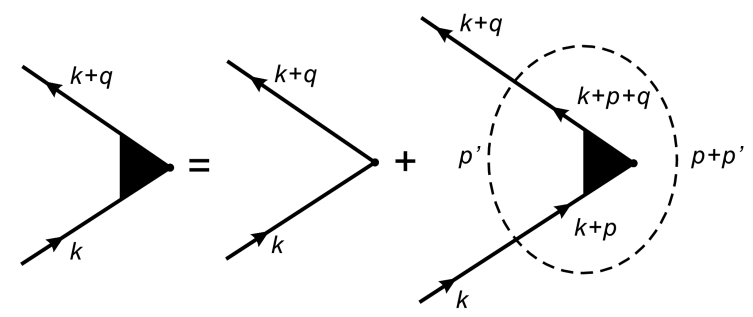

Fig. 6. Equation for the dressed vertex function employing the ladder-type approximation. The solid lines represent the charge carrier Green functions in the Nambu notation, and the dashed ones belong to the spin-bubble dressing of the charge carriers [10, 14].

In order to prove that the approximation (6) for the dressed vertex in fact implies a gauge invariant description of the Meissner effect, it is necessary and sufficient to check whether it does not violate the GWI. The proof is a three-step procedure [21]: first we check that the GWI for the bare current vertex is satisfied with the mean-field charge carrier Green function [10, 14]. Then, in the second step, the GWI is written with the dressed current vertex satisfying the ladder-type vertex equation in Fig. 6. Eventually, the GWI for the mean-field charge carrier Green function is employed, along with the equation for the full (dressed) charge carrier Green function including the spin-bubble self-energy. This yields the GWI (and hence ensures gauge invariance) for the ladder-like dressed vertex and dressed Green function. Consequently, the kernel of the response function with the paramagnetic contribution calculated from the dressed polarization bubble shown in Fig. 5 is gauge invariant.

\section{References}

[1] W.N. Hardy, D.A. Bonn, D.C. Morgan, R. Liang, K. Zhang, Phys. Rev. Lett. 70, 3999 (1993).

[2] A. Damascelli, Z. Hussain, Z.X. Shen, Rev. Mod. Phys. 75, 473 (2003).

[3] S. Kamal, R. Liang, A. Hosseini, D.A. Bonn, W.N. Hardy, Phys. Rev. B 58, R8933 (1998).

[4] D.M. Broun, W.A. Huttema, P.J. Turner, S. Özcan, B. Morgan, R. Liang, W.N. Hardy, D.A. Bonn, Phys. Rev. Lett. 99, 237003 (2007).

[5] Y. Uemura, G.M. Luke, B.J. Sternlieb, J.H. Brewer, J.F. Carolan, W.N. Hardy, R. Kadono, J.R. Kempton, R.F. Kiefl, S.R. Kreitzman, P. Mulhern, T.M. Riseman, D.Ll. Williams, B.X. Yang, S. Uchida, H. Takagi, J. Gopalakrishnan, A.W. Sleight, M.A. Subramanian, C.L. Chien, M.Z. Cieplak, Phys. Rev. Lett. 62, 2317 (1989); Y. Uemura, L.P. Le, G.M. Luke, B.J. Sternlieb, W.D. Wu, J.H. Brewer, T.M. Riseman, C.L. Seaman, M.B. Maple, M. Ishikawa, D.G. Hinks, J.D. Jorgensen, G. Saito, H. Yamochi, Phys. Rev. Lett. 66, 2665 (1991).

[6] R. Khasanov, D.G. Eshchenko, H. Luetkens, E. Morenzoni, T. Prokscha, A. Suter, N. Garifianov, M. Mali, J. Roos, K. Conder, H. Keller, Phys. Rev. Lett. 92, 057602 (2004).

[7] A. Suter, E. Morenzoni, R. Khasanov, H. Luetkens, T. Prokscha, N. Garifianov, Phys. Rev. Lett. 92, 087001 (2004).

[8] I. Kosztin, A.J. Leggett, Phys. Rev. Lett. 79, 135 (1997).

[9] Shiping Feng, Phys. Rev. B 68, 184501 (2003); Shiping Feng, Tianxing Ma, Huaiming Guo, Physica $C$ 436, 14 (2006).

[10] See, e.g., the review: Shiping Feng, Huaiming Guo, $\mathrm{Yu}$ Lan, Li Cheng, Int. J. Mod. Phys. B 22, 3757 (2008), and references therein.

[11] D.E. Sheehy, T.P. Davis, M. Franz, Phys. Rev. B 70 , 054510 (2004).

[12] P.W. Anderson, in: Frontiers and Borderlines in Many Particle Physics, Eds. R.A. Broglia, J.R. Schrieffer, North-Holland, Amsterdam 1988, p. 1; Science 235, 1196 (1987).

[13] K.A. Chao, J. Spałek, A.M. Oleś, J. Phys. C 10, L271 (1977). 
[14] Shiping Feng, Jihong Qin, Tianxing Ma, J. Phys., Condens. Matter 16, 343 (2004).

[15] P. Wróbel, R. Eder, R. Micnas, J. Phys., Condens. Matter 15, 2755 (2003); P. Wróbel, R. Eder, P. Fulde, J. Phys., Condens. Matter 15, 6599 (2003); P. Wróbel, A. Maciąg, R. Eder, P. Fulde, R. Micnas, Acta Phys. Pol. A 106, 575 (2004).

[16] H. Eskes, A.M. Oleś, M.B.J. Meinders, W. Stephan, Phys. Rev. B 50, 17980 (1994).

[17] A.L. Fetter, J.D. Walecka, Quantum Theory of Many-Particle Systems, McGraw-Hill, New York 1971, Sect. 13.52 .

[18] H. Fukuyama, H. Ebisawa, Y. Wada, Prog. Theor. Phys. 42, 494 (1969); H. Fukuyama, Prog. Theor. Phys. 42, 1284 (1969).

[19] M. Krzyzosiak, R. Gonczarek, Physica C 426-431, 273 (2005); Int. J. Mod. Phys. B 18, 4143 (2004); R. Gonczarek, M. Krzyzosiak, in: Progress in Superconductivity Research, Ed. O.A. Chang, Nova Science Publishers, New York 2008, p. 163.

[20] M. Krzyzosiak, Yu Lan, Shiping Feng, R. Gonczarek, arXiv:09040093.
[21] J.R. Schrieffer, Theory of Superconductivity, Addison-Wesley, Reading, MA 1964.

[22] M. Tinkham, Introduction to Superconductivity, McGraw-Hill, New York 1996, App. 3.

[23] See, e.g., B.A. Bonn, W.N. Hardy, in: Physical Properties of High Temperature Superconductors $V$, Ed. D.M. Ginsberg, World Scientific, Singapore 1996, and references therein.

[24] T. Pereg-Barnea, P.J. Turner, R. Harris, G.K. Mullins, J.S. Bobowski, M. Raudsepp, R. Liang, D.A. Bonn, W.N. Hardy, Phys. Rev. B 69, 184513 (2004); C. Panagopoulos, J.R. Cooper, G.B. Peacock, I. Gameson, P.P. Edwards, W. Schmidbauer, J.W. Hodby, Phys. Rev. B 53, R2999 (1996).

[25] S. Misawa, Phys. Rev. B 49, 6305 (1994).

[26] P.I. Arseev, S.O. Loiko, N.K. Fedorov, Phys.-Usp. 49, 1 (2006). 